\title{
An Analytical Scattering Model for Low Energy Annular Dark Field Transmission Scanning Electron Microscopy
}

Taylor Woehl, ${ }^{1}$ Jason Holm, ${ }^{1}$ and Robert Keller $^{1}$

1. Material Measurement Lab, NIST, Boulder, CO 80305

Scanning transmission electron microscopy (STEM) is a powerful tool for chemical and structural analysis of materials on the nano- and atomic-scale. Often the atomic scale resolution afforded by aberration corrected STEM is not needed, and in some cases the high beam currents and electron energies used can damage composite nanomaterials containing organic components. A relatively new approach for STEM imaging when atomic resolution is not required is STEM imaging in a scanning electron microscopy (SEM) equipped with a transmission detector (t-SEM) [1-3]. This approach has the advantage of being a comparatively easy to use and low cost solution for performing STEM imaging with a resolution of several nanometers. While the increased amount of scattering by the lower-energy electrons (typically $30 \mathrm{keV}$ ) can increase signals for imaging and spectroscopy, it also leads to artifacts in samples on thick substrates, such as contrast inversion, low signal-to-noise, and convoluted image contrast [1-3]. Monte Carlo simulations have predicted contrast of annular dark field (ADF) t-SEM images of nanoparticles on thick layers of carbon support material [1]; however, the models did not give much insight into the physical mechanisms leading to the contrast. An analytical model to predict image contrast would help to identify imaging conditions that are void of imaging artifacts and optimize contrast and resolution.

Here we employ an analytical electron scattering model to show that for decreasing collection angles, the decrease in ADF t-SEM contrast of gold nanoparticles on carbon films is consistent with increased thickness contrast from the carbon substrate at low scattering angles. Images of gold nanoparticles on lacey carbon TEM grids were acquired in an SEM (30 keV) with an ADF detector masked by an annular aperture to define inner and outer collection angles. We varied the distance between the sample and detector to systematically vary the ADF collection angles and found that the contrast of the gold nanoparticles decreased as a function of the ADF inner collection angle (Figure 1a). Nanoparticle contrast (Figure 1b) was lower overall for particles on the thicker lacey carbon support. To model the experimental contrast, we developed an analytical electron scattering model that includes collection angle-dependent elastic inelastic, and thermal diffuse electron scattering as well as geometric considerations for the ADF detector. We calculated the contrast by taking the difference between the number of electrons transmitted through the nanoparticle and carbon substrate to a solid angle defined by the inner and outer collection angles. The theoretical contrast was compared to the experimental images using the carbon support thickness as an adjustable parameter, as we could not accurately determine this thickness. With this fitting parameter, there was good agreement between the model predictions and experimental contrast (Figure 1c). The model indicated that the decrease in contrast with decreasing collection angle was due to increased thickness contrast from the carbon support, relative to the atomic number contrast of the gold nanoparticles. The discrepancy in the carbon support thickness 
is likely due to multiple scattering in the nanoparticles. We expect this analytical scattering model will be important for determining artifact-free imaging conditions for ADF t-SEM imaging [5].

\section{References:}

[1] N. Brodusch, H. Demers, R. Gauvin, Microscopy and Microanalysis, 19 (2013) 1688-1697.

[2] V. Morandi, P.G. Merli, Journal of Applied Physics 101 (2007), 114917.

[3] B. Patel, M. Watanabe, Microscopy and Microanalysis 20 (2014), 124-132.

[4] T. Klein, E. Buhr, C.G. Frase, in: P.W. Hawkes (Ed.) Advances in Imaging and Electron Physics, 171 (2012), 297-356.

[5] T. Woehl and J. Holm acknowledge funding from the National Research Council Postdoctoral Research Associateship Program. Contribution of the U.S. Department of Commerce; not subject to copyright in the United States.

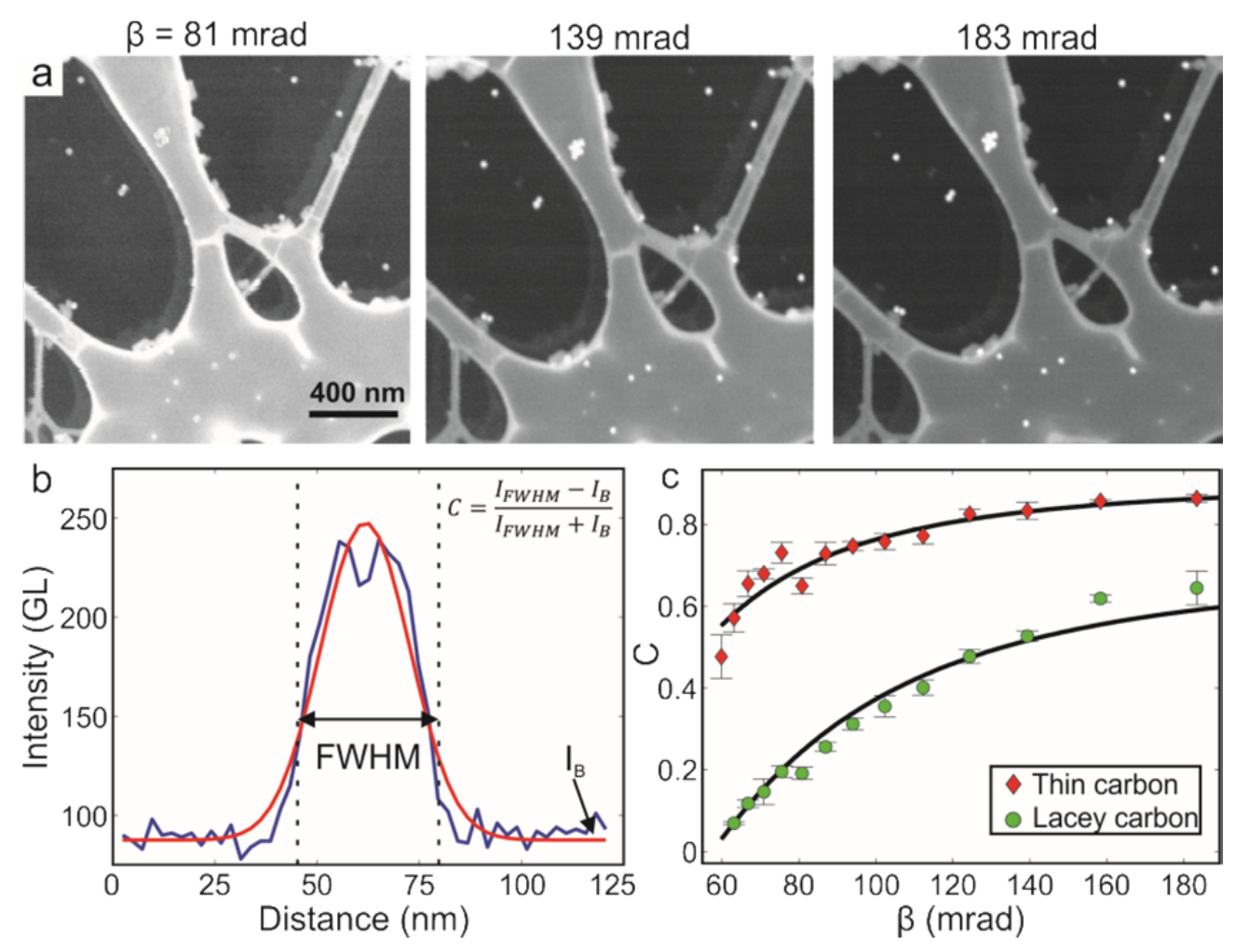

Figure 1. (a) Experimental ADF t-SEM images of $30 \mathrm{~nm}$ gold nanoparticles on a lacey carbon TEM grid at various inner collection angles. (b) Method for experimental measurement of the contrast of gold nanoparticles on the TEM grid. The contrast is equal to the difference of the mean intensity inside the full width half maximum $\left(I_{F W H M}\right)$ of the nanoparticle and the background intensity, divided by the total intensity. (c) Experimental and theoretical contrast for nanoparticles on thin and lacey carbon. Each data point is the mean contrast of 5 nanoparticles. Error bars represent two standard deviations of the mean. The black lines are the theoretically determined contrast using substrate thicknesses of 85 and 300 $\mathrm{nm}$ for the thin and lacey carbon, respectively. 